\title{
A Descriptive Algorithm for Sobel Image Edge Detection
}

\author{
O. R. Vincent \\ Clausthal University of \\ Technology, Germany \\ and University of Agriculture, \\ Abeokuta, Nigeria
}

vincent.rebecca@gmail.com

\author{
O. Folorunso \\ Department of Computer \\ Science, \\ University of Agriculture, \\ Abeokuta, Nigeria
}

folorunsolusegun@yahoo.com

\begin{abstract}
Image edge detection is a process of locating the edge of an image which is important in finding the approximate absolute gradient magnitude at each point $I$ of an input grayscale image. The problem of getting an appropriate absolute gradient magnitude for edges lies in the method used. The Sobel operator performs a 2-D spatial gradient measurement on images. Transferring a 2-D pixel array into statistically uncorre lated data set enhances the removal of redundant data, as a result, reduction of the amount of data is required to represent a digital image. The Sobel edge detector uses a pair of $3 \times 3$ convolution masks, one estimating gradient in the $\mathrm{x}$-direction and the other estimating gradient in $y$-direction. The Sobel detector is incredibly sensitive to noise in pictures, it effectively highlight them as edges. Hence, Sobel operator is recommended in massive data communication found in data transfer.
\end{abstract}

Ke ywords: Image Processing, Edge Detection, Sobel Operator, Data Communication and Absolute Gradient Magnitude.

\section{Introduction}

Image processing is important in modern data storage and data transmission especially in progressive transmission of images, video coding (teleconferencing), digital libraries, and image database, remote sensing. It has to do with manipulation of images done by algorithm to produce desired images (Milan et al., 2003). Digital Signal Processing (DSP) improve the quality of images taken under extremely unfavourable conditions in several ways: brightness and contrast adjustment, edge detection, noise reduction, focus adjustment, motion blur reduction etc (Gonzalez, 2002). The advantage is that image processing allows much wider range of algorithms to be applied to the input data in order to avoid problems such as the build-up of noise and signal distortion during processing (Baker \& Nayar, 1996). Many of the techniques of digital image processing were developed in the 1960's at the Jet Propulsion Laboratory, Massachusetts Institute of

Material published as part of this publication, either on-line or in print, is copy righted by the Informing Science Institute. Permission to make digital or paper copy of part or all of these works for personal or classroom use is granted without fee provided that the copies are not made or distributed for profit or commercial advantage AND that copies 1) bear this notice in full and 2) give the full citation on the first page. It is permissible to abstract these works so long as cred it is given. To copy in all other cases or to republish or to post on a server or to redistribute to lists requires specific permission and payment of a fee. Contact Publisher@InformingScience.org to request redistribution permission.
Technology (MIT), Bell laboratory and few other places. But the cost of processing was fairly high with the computing equipments of that era.

With the fast computers and signal processors available in the 2000's, digital image processing became the most common form of image processing and is general used because it is not only the most versatile method but also the 
cheapest. The process allows the use of much more complex algorithms for image processing and hence can offer both more sophisticated performance at simple tasks, and the implementation of methods which would be impossible by analog means (Micheal, 2003). Thus, images are stored on the computers as collection of bits representing pixel or points forming the picture elements (Vincent, 2006). Firstly, images are a measure of parameter over space, while most signals are measures of parameter over time. Secondly, they contain a great deal of information (Guthe \& Strasser, 2004); image processing is any form of information processing for which the input is an image, such as frames of video; the output is not necessarily an image, but can be for instance be a set of features of the image (Yuval, 1996).

Most image-processing techniques involve treating the image as a two-dimensional signal and applying standard signal-processing techniques to it. The process involves the enhancement or manipulation of an image which resulted in another image, removal of redundant data and the transformation of a 2-D pixel array into a statically uncorre lated data set (Priotr, 2004). Since images contain lots of redundant data, scholars have discovered that the most important information lies in it edges (Canny, 1986). Edges being the local property of a pixel and its immediate neighbourhood, characterizes boundary (Chaug-Huang, 2002). They correspond to object boundaries, changes in surface orientation and describe defeat by a small margin. Edges typically correspond to points in the image where the gray value changes significantly from one pixel to the next. Edges represents region in the image with strong intensity contrast; representing an image by its edges has the fundamental advantage that the amount of data is reduced significantly while retaining most of image's vital information with high frequencies (Keren, Osadchy, \& Gotsman, 2001). Thus, detecting Edges help in extracting useful information characteristics of the image where there are abrupt changes (Folorunso et al., 2007).

Edge detection is a process of locating an edge of an image. Detection of edges in an image is a very important step towards understanding image features. Edges consist of meaningful features and contained significant information. It's reduce significantly the amount of the image size and filters out information that may be regarded as less relevant, preserving the important structural properties of an image (Yuval, 1996). Most images contain some amount of redundancies that can sometimes be removed when edges are detected and replaced, when it is reconstructed (Osuna et al., 1997). Eliminating the redundancy could be done through edge detection. When image edges are detected, every kind of redundancy present in the image is removed (Sparr, 2000).

The purpose of detecting sharp changes in image brightness is to capture important events. Applying an edge detector to an image may significantly reduce the amount of data to be processed and may therefore filter out information that may be regarded as less relevant, while preserving the important structural properties of an image. The image quality reflects significant information in the output edge and the size of the image is reduced. This in turn explains further that edge detection is one of the ways of solving the problem of high volume of space images occupy in the computer memory. The problems of storage, transmission over the Internet and bandwidth could be solved when edges are detected (Vincent, 2007). Since edges often occur at image locations representing object boundaries, edge detection is extensively used in image segmentation when images are divided into areas corresponding to different objects.

\section{Related Methods}

Different methods are used to detect edges in image processing among these is Roberts Cross Algorithms. Robert process a photograph into a line drawing, transform the line drawing into a three-dimensional representation and finally display the three-dimensional structure with all the hidden lines removed, from any point of view (Robert, 1965). The Roberts cross algorithm (Mario\& Maltoni, 1997) performs a 2-D spatial gradient convolution on the image. The main idea is to bring out the horizontal and vertical edges individually and then to put them together for the 
resulting edge detection. The two filters highlight areas of high special frequency, which tend to define the edge of an object in an image. The two filters are designed with the intention of bringing out the diagonal edges within the image. The $G x$ image will enunc iate diagonals that run from thee top-left to the bottom-right where as the $G y$ image will bring out edges that run topright to bottom-left. The two individual images $G x$ and $G y$ are combined using the approximation equation $|G|=|G x|+|G y|$

The Canny edge detection operator was developed by John F. Canny in 1986 and uses a multistage algorithm to detect a wide range of edges in images. In addition, canny edge detector is a complex optimal edge detector which takes significantly longer time in result computations. The image is firstly run through a Gaussian blur to get rid of the noise. When the algorithm is applied, the angle and magnitude is obtained which is used to determine portions of the edges to retain. There are two threshold cut-off points where any value in the image below the first threshold is dropped to zero and values above the second threshold is raised to one.

Canny (1986) considered the mathematical problem of deriving an optimal smoothing filter given the criteria of detection, localization and minimizing multiple responses to a single edge. $\mathrm{He}$ showed that the optimal filter given these assumptions is a sum of four exponential terms. He also showed that this filter can be well approximated by first-order derivatives of Gaussians. Canny also introduced the notion of non-maximum suppression, which means that given the presmoothing filters, edge points are defined as points where the gradient magnitude assumes a local maximum in the gradient direction.

Another algorithm used is the Susan edge detector. This edge detection algorithm follows the usual method of taking an image and using a predetermined window centered on each pixel in the image applying a locally acting set of rules to give an edge response (Vincent, 2006). The response is then processed to give the output as a set of edges. The Susan edge filter has been implemented using circular masks (kernel) to give isotopic responses with approximations used either with constant weighting within it or with Gaussian weighting. The usual radius is 3.4 pixels, giving a mask of 37 pixels, and the smallest mask considered is the traditional $3 \times 3$ mask. The 37 pixels circular mask used in all feature detection experiments is placed at each point in the image and for each point the brightness of each pixel within the mask is compared with that of nucleus. The comparison equation is

$$
C\left(\stackrel{r}{r}, r_{o}^{r}\right)=\left\{\begin{array}{lll}
1 & \text { if } & \left|\left(\begin{array}{l}
\mathrm{I} \\
r
\end{array}\right)-\mathrm{I}\left(\mathrm{r}_{o}\right)\right| \leq t \\
0 & \text { if } & \left|\mathrm{I}(\stackrel{\mathrm{r}}{r})-\mathrm{I}\left(\mathrm{r}_{o}\right)\right|>t
\end{array}\right.
$$

where $r$ is the position of the nucleus in the dimensional image, $r_{o}$ is the position of any other point within the mask, $I(r)$ is the brightness of any pixel, $t$ is the brightness in difference threshold and $C$ is the output of the comparison. This comparison is done for each pixel within the mask where total $n$ of the outputs $(c)$ is given as

$$
\mathrm{n}\left(\dot{r}_{o^{\circ}}\right)=\sum_{r} C\left(\begin{array}{ll}
\mathrm{I} & \dot{r}_{o}
\end{array}\right)
$$




\section{Sobel Filter Design}

Most edge detection methods work on the assumption that the edge occurs where there is a discontinuity in the intensity function or a very steep intensity gradient in the image. Using this assumption, if one take the derivative of the intensity value across the image and find points where the derivative is maximum, then the egde could be located. The gradient is a vector, whose components measure how rapid pixel value are changing with distance in the $x$ and $y$ direction. Thus, the components of the gradient may be found using the following approximation:

$$
\begin{aligned}
& \frac{\partial f(x, y)}{\partial x}=\Delta x=\frac{f(x+d x, y)-f(x, y)}{d x} \\
& \frac{\partial f(x, y)}{\partial x}=\Delta y=\frac{f(x, y+d y)-f(x, y)}{d y}
\end{aligned}
$$

where $d x$ and $d y$ measure distance along the $x$ and $y$ directions respectively. In discrete images, one can consider $d x$ and $d y$ in terms of numbers of pixel between two points. $d x=d y=1$ (pixel spacing) is the point at which pixel coordinates are $(i, j)$ thus,

$$
\begin{gathered}
\Delta x=f(i+1, j)-f(i, j) \\
\Delta y=f(i, j+1)-f(i, j)
\end{gathered}
$$

In order to detect the presence of a gradient discontinuity, one could calculate the change in the gradient at $(i, j)$.This can be done by finding the following magnitude measure

$$
M=\sqrt{\Delta x^{2}+\Delta^{2} y}
$$

and the gradient direction $\theta$ is given by

$$
\theta=\tan ^{-1}\left[\frac{\Delta y}{\Delta x}\right]
$$

\section{Method of the Filter Design}

There are many methods of detecting edges; the majority of different methods may be grouped into these two categories:

i. Gradient: The gradient method detects the edges by looking for the maximum and minimum in the first derivative of the image. For example Roberts, Prewitt, Sobel where detected features have very sharp edges. (see Figure 1)

ii. Laplacian: The Laplacian method searches for zero crossings in the second derivative of the image to find edges e.g. Marr-Hildreth, Laplacian of Gaussian etc. An edge has onedimensional shape of a ramp and calculating the derivative of the image can highlight its location (see Figure 2).

Edges may be viewpoint dependent: these are edges that may change as the viewpoint changes and typically reflect the geometry of the scene which in turn reflects the properties of the viewed objects such as surface markings and surface shape. A typical edge might be the border between a block of red colour and a block of yellow, in contrast. However, what happens when one looks at the pixels of that image is that all visible portion of one edge are compacted. 


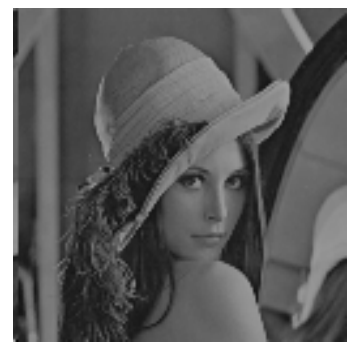

Input Image

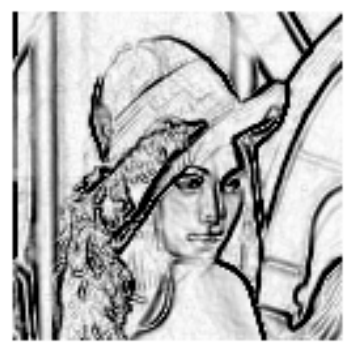

Output Edges

Figure 1: The Gradient Method

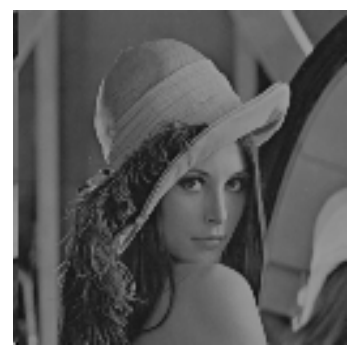

Input Image

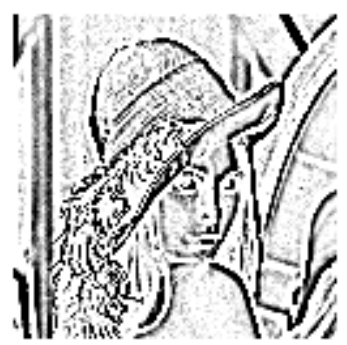

Output Edges

Figure 2: the Laplacian Method

The Sobel operator is an example of the gradient method. The Sobel operator is a discrete differentiation operator, computing an approximation of the gradient of the image intensity function (Sobel \& Feldman, 1968). The different operators in eq. (5) and (6) correspond to convolving the image with the following marks

$$
\Delta x=\left[\begin{array}{cc}
-1 & 1 \\
0 & 0
\end{array}\right], \Delta y=\left[\begin{array}{cc}
-1 & 0 \\
1 & 0
\end{array}\right]
$$

When this is done, then:

i. The top left-hand corner of the appropriate mask is super-imposed over each pixel of the image in turn,

ii. A value is calculated for $\Delta x$ or $\Delta y$ by using the mask coefficients in a weighted sum of the value of pixels $(i, j)$ and its neighbours,

iii. These masks are referred to as convolution masks or sometimes convolution kernels. Instead of finding approximate gradient components along the $x$ and $y$ directions, approximation of the gradient components could be done along directions at $45^{\circ}$ and $135^{\circ}$ to the axes respectively. In this case

$$
\begin{aligned}
\Delta x & =f(i+1, j+1)-f(i, j) \\
\Delta y & =f(i, j+1)-f(i+1, j)
\end{aligned}
$$

This form of operator is known as the Roberts edge operator and was one of the first set of operators used to detect edges in images (Robert, 1965). The corresponding convolution masks are given by: 


$$
\Delta_{1}=\left[\begin{array}{cc}
0 & 1 \\
-1 & 0
\end{array}\right] \text { and } \Delta_{2}=\left[\begin{array}{cc}
1 & 0 \\
0 & -1
\end{array}\right]
$$

An advantage of using a larger mask size is that the errors due to the effects of noise are reduced by local averaging within the neighbourhood of the mask. An advantage of using a mask of odd size is that the operators are centered and can therefore provide an estimate that is based on a center pixel $(i, j)$. One important edge operator of this type is the Sobel edge operator. The Sobel edge operator masks are given as

$$
\Delta x=\left[\begin{array}{lll}
-1 & 0 & 1 \\
-2 & 0 & 2 \\
-1 & 0 & 1
\end{array}\right] \quad \Delta y=\left[\begin{array}{ccc}
1 & 2 & 1 \\
0 & 0 & 0 \\
-1 & -2 & -1
\end{array}\right]
$$

The operator calculates the gradient of the image intensity at each point, giving the direction of the largest possible increase from light to dark and the rate of change in that direction. The result therefore shows how "abruptly" or "smoothly" the image changes at that point and therefore how likely it is that part of the image represents an edge, as well as how that the edge is likely to be oriented. In practice, the magnitude (likelihood of an edge) calculation is more reliable and easier to interpret than the direction calculation. Mathematically, the gradient of a two-variable function (the image intensity function) at each image point is a $2 \mathrm{D}$ vector with the components given by the derivatives in the horizontal and vertical directions. At each image point, the gradient vector points to the direction of largest possible intensity increase, and the length of the gradient vector corresponds to the rate of change in that direction. This implies that the result of the Sobel operator at any image point which is in a region of constant image intensity is a zero vector and at a point on an edge is a vector which points across the edge, from darker to brighter values. The algor ithm for developing the Sobel model for edge detection is given below.

\section{Pseudo-codes for Sobel edge detection method}

Input: A Sample Image

Output: Detected Edges

Step 1: Accept the input image

Step 2: Apply mask $G x, G y$ to the input image

Step 3: Apply Sobel edge detection algorithm and the gradient

Step 4: Masks manipulation of $G x, G y$ separately on the input image

Step 5: Results combined to find the absolute magnitude of the gradient

$$
|G|=\sqrt{G x^{2}+G y^{2}}
$$

Step 6: the absolute magnitude is the output edges 


\section{Second order derivative operators}

A maximum of the first derivative will occur at a zero crossing of the second derivative. To get both horizontal and vertical edges, we look at second derivative in both the $x$ and $y$ directions. This is the Laplacian of $I$ where

$$
\nabla^{2} I=\frac{\partial^{2} I}{\partial x^{2}}+\frac{\partial^{2} I}{\partial y^{2}}
$$

The Laplacian is linear and rotationally symmetric. Thus, if one search for the zero crossing of the image that is first smoothed with a Gaussian mask and then the second derivative is calculated; or one can convolve the image with the Laplacian of the Gaussian also known as the LoG operator.

$$
\nabla^{2}(G \otimes \mathrm{I})=\nabla^{2} G \otimes \mathrm{I}
$$

The edge is modeled by specifying its four degrees of freedom: its position, its orientation, and the constant intensities on either side of the step. The data is matched by seeking the least squares error fit of the parametric model to the image window but such an approach is generally and computationally expensive. Normally what is done is that both the image data and the model are represented over small windows by their first derivative coefficients in a particular 2-D orthonormal series expansion. In this case the optimization reduces to just one variable: the orientation of the edge.

\section{Results and Discussion}

The Sobel operator performs a 2-D spatial gradient measurement on an image. Typically, it is used to find the approximate absolute gradient magnitude at each point $I$ of an input grayscale image. The Sobel edge detector uses a pair of $3 \times 3$ convolution masks, one estimating gradient in the $x$-direction and the other estimating gradient in $y$-direction. A convolution is usually much smaller than the actual image. As a result, the mask is slide over the image manipulating a square of pixels at a time. The mask is slides over an area where the input image changes with that pixel's value and then shifts one pixel to the right and continues to the right until it reaches the end of the row which automatically starts again at the beginning of the next row. It is important to note that pixels in the first row and last row, as well as the first and last column cannot be manipulated by a $3 \times 3$ mask.

This is because when placing the centre of the mask over a pixel in the first row for example, the mask will be outside the image boundaries. The $G_{x}$ mask highlights the edges in the horizontal direction while the $G_{y}$ mask highlights the edges in vertical direction. After taking the magnitude of both, the resulting output detects edges in both directions. This is done by:

(1) Applying noise smoothing to the original image

(2) Filtering the original image following two kernels gives the result in Table 1.

Table 1: Filte ring Results of the two Kernel

\begin{tabular}{|l|l|l|l|l|l|}
\hline \multicolumn{2}{|c|}{ Kernel1=Gx } & \multicolumn{3}{c|}{ Kernel 2 = Gy } \\
\hline-1 & 0 & 1 & -1 & -2 & -1 \\
\hline-2 & 0 & 2 & 0 & 0 & 0 \\
\hline-1 & 0 & 1 & 1 & 2 & 1 \\
\hline
\end{tabular}


Obtaining $I_{1}$ and $I_{2}$

(3) Estimating the gradient magnitude at each pixel as

$$
G(i, j)=\sqrt{\left(I_{1}\right)^{2}+\left(I_{2}\right)^{2}}
$$

(4) Marking the pixel as edge points if $G(i, j)>\tau$ results the image in Figure 3

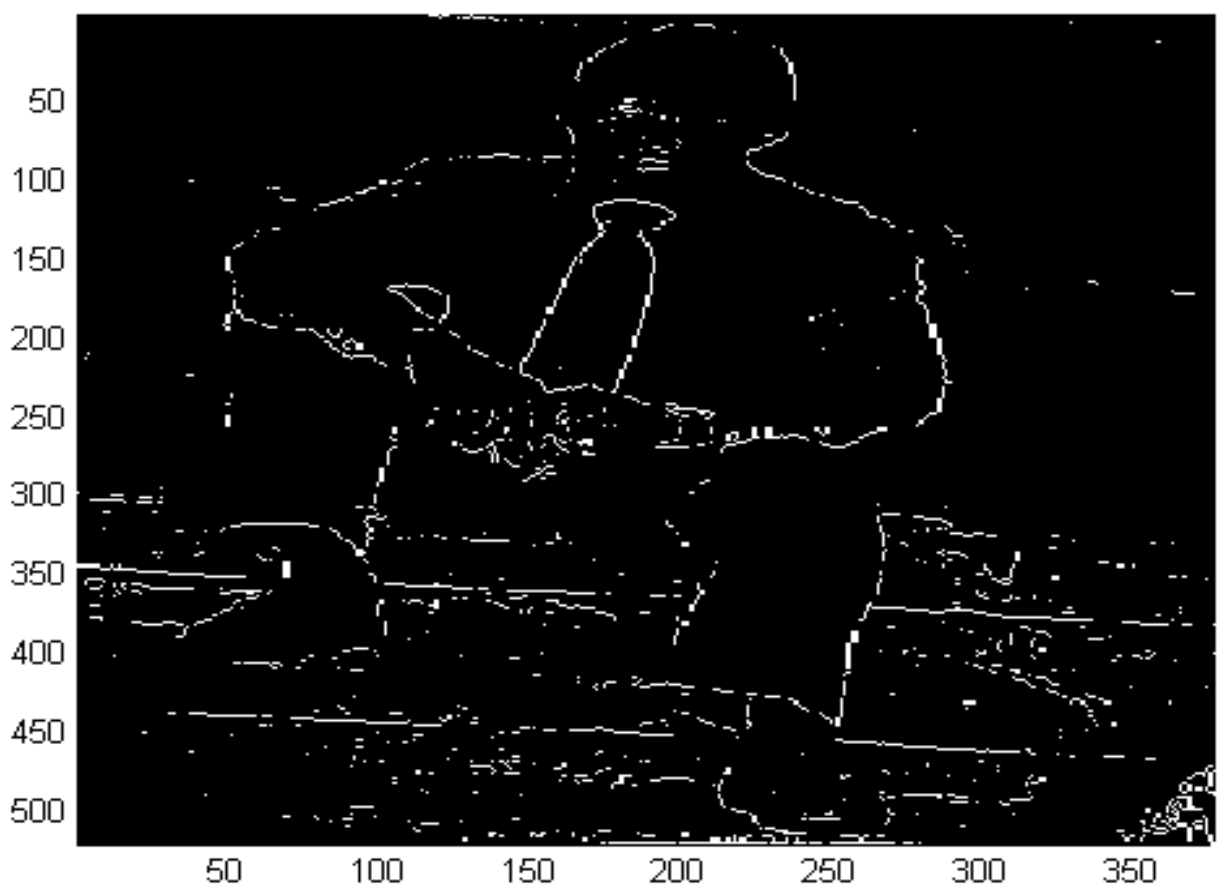

Figure 3: The Detected Image Edge

\section{Practical Implications and Importance of Edge Detection}

The following advantages of Sobel edge detector justify its superiority over other edge detection techniques:

i. Edge Orientation: The geometry of the operator determines a characteristic direction in which it is most sensitive to edges. Operators can be optimized to look for horizontal, vertical, or diagonal edges.

ii. Noise Environment: Edge detection is difficult in noisy images, since both the noise and the edges contain high-frequency content. Attempts to reduce the noise result in blurred and distorted edges. Operators used on noisy images are typically larger in scope, so they can average enough data to discount localized noisy pixels. This results in less accurate localization of the detected edges.

iii. Edge Structure: Not all edges involve a step change in intensity. Effects such as refraction or poor focus can result in objects with boundaries defined by a gradual change in intensity. The operator is chosen to be responsive to such a gradual change in those cases. Newer wavelet-based techniques actually characterize the nature of the transition for each 
edge in order to distinguish, for example, edges associated with hair from edges associated with a face.

Edges play quite an important role in many applications of image processing, in particular for machine vision systems that analyze scenes of man-made objects under controlled illumination conditions. Detecting edges of an image represents significantly reduction the amount of data and filters out useless information, while preserving the important structural properties in an image. Hence, edge detection is a form of knowledge management.

\section{Conclusion}

The Sobel operator performs a 2-D spatial gradient measurement on an image. Typically it is used to find the approximate absolute gradient magnitude at each point $I$ of an input grayscale image. The Sobel edge detector uses a pair of $3 \times 3$ convolution masks, one estimating gradient in the $x$ direction and the other estimating gradient in $y$-direction. It is easy to implement than the other operators. Transferring a 2-D pixel array into statistically uncorrelated data set enhances the removal of redundant data, as a result, reduction of the amount of data required to represent a digital image. Considering data communication especially the internet, massive data transfer causes serious problems for interactive network users.

Edge detection helps in optimizing network bandwidth and it is needed to keep track of data flowing in and out of the network. It helps to extract useful features for pattern recognition. Although the Sobel operator is slower to compute, it's larger convolution kernel smoothes the input image to a greater extent and so makes the operator less sensitive to noise. The larger the width of the mask, the lower its sensitivity to noise and the operator also produces considerably higher output values for similar edges. Sobel operator effectively highlights noise found in real world pictures as edges though, the detected edges could be thick. The Canny edge detector and similar algorithm solved these problems by first blurring the image slightly then applying an algorithm that effectively thins the edges to one-pixel. This may constitute a much slower process, hence, Sobel operator is highly recommended in massive data communication found in image data transfer.

The Sobel operator is based on convolving the image with a small, separable, and integer valued filter in horizontal and vertical direction and is therefore relatively inexpensive in terms of computations. On the other hand, the gradient approximation which it produces is relatively crude, in particular for high frequency variations in the image.

\section{References}

Agaian, S. S., Baran, T. A., \& Panetta, K. A. (2003). Transform-based image compression by noise reduction and spatial modification using Boolean minimization. IEEE Workshop on Statistical Signal Processing, 28 Sept.-1 Oct. pp. $226-229$.

Baker, S., \& Nayar, S. K. (1996). Pattern rejection. Proceedings of IEEE Conference Computer Vision and Pattern Recognition, 544-549.

Canny, J. F. (1986). A computational approach to edge detection. IEEE Trans Pattern Analysis and Machine Intelligence, 8(6), 679-698.

Chang-Huang, C. (2002). Edge detection based on class ratio. 152, sec.3, Peishen Rd., Shenkeng, Taipei, 22202, Taiwan, R.O.C.

Folorunso O., Vincent, O. R., \& Dansu, B. M. (2007). Image edge detection: A knowledge management technique for visual scene analysis. Information Management and Computer Security, 15(1), 23-32.

Gon zalez, R., \& Woods, R. (2002). Digital image processing (2nd ed.). Prentice-Hall Inc. 567-612. 
Guthe, S., \& Strasser, W. (2004). Advanced techniques for high-quality multi-resolution volume rendering. Graphics and Visualization, 28, 25-78.

Keren, D., Osadchy, M., \& Gotsman. C. (2001). Antifaces: A novel, fast method for image detection. IEEE Transactions on Pattern Analysis and Machine Intelligence, 23(7), 747-761.

Mario, D., \& Maltoni, D. (1997). Direct gray-scale minutiae detection in fingerprints. IEEE Transactions on Pattern Analysis and Machine Intelligence, 19(1), 27-40.

Michael, U. (2003). Mathe matics properties of the JPEG 2000 wavelet filters. IEEE Transactions on Image Processing, 12(9), 080-1090.

Milan, S., Vaclav, H., \& Roger, B. (2002). Image processing analysis and machine vision. London: Chapman and Hall, 255-280.

Osuna, E., Freund, R., \& Girosi, F. (1997). Training support vector machines: An application to face detection. Proceedings of IEEE Conference Computer Vision and Pattern Recognition.

Priotr, P. (2004). Practical signal decomposition design based on Haar - Wavelet transformation. Journal of Applied Computer Science, 2(1), 61-82.

Roberts, L. G. (1965). Machine perception of three-dimensional solids. In J. T. Tippett (Ed.), Optical and electro-optical information processing (Ch. 9, pp. 159-197). Cambridge, Massachusetts: MIT Press.

Scharr, H. (1996). Digitale Bildverarbeitung und Papier: Texturanalyse mittels Pyramiden und Grauwertstatistiken am Beispiel der Papierformation. Master's thes is, Faku ltÄat fÄur Physik und Astronomie, Ruprecht-Karls-UniversitÄat Heidelberg, Germany.

Sobel, I., \& Feld man, G. (1968). A $3 \times 3$ isotropic gradient operator for image processing. Presented at a talk at the Stanford Artificial Project.

Sparr, G. (2002). Image processing and pattern classification for character recognition. Center for Mathematical Sciences, Lund University, 2, 25-78.

Vincent, O. R., Folorunso, O., \& Agboola, A. A. A. (2006). Modified algorith m for transform-based image compression. Journal of Computer Science and its Application, 13(1), 43-48.

Vincent, O. R. (2007).Optimization of network bandwidth using image compression. Proceedings of the National Conference on advanced Data Computing Communications and Security, Kadi, India.

Yuval, F. (1996). Fractal image compression (theory and application). Institute for non-linear Science, University of California, San Diego, USA. 


\section{Biographies}

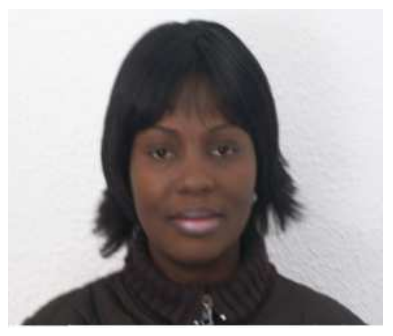

Rebecca Vincent is a Lecturer in the Department of Computer Science, University of Agriculture, Abeokuta. She obtained a B.Sc degree in Mathematical Sciences (Computer Science Option) and M.Sc in Computer Science from the University of Agriculture, Abeokuta in 2000 and 2005 respectively. She is currently on her Ph.D and studies now with the Computational Intelligence Group, at the Institute of Informatics, Clausthal University of Technology, Germany; where she carries out research on Mobile Agents for E-Commerce. Her research interest include: Images and Vision, Know ledge Management, Computational Complexity, Ecommerce, Agents and Mobile Agents. She is a member of Nigeria Computer Soc iety and has published in notable International and local Journals.

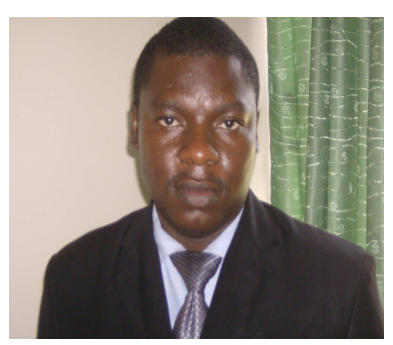

Dr. Olusegun Folorunso is a Senior Lecturer in the Department of Computer Science, University of Agriculture, Abeokuta. He obtained a B.Sc degree in Mathematical Sciences from the University of Agriculture, Abeokuta in 1992, M.Sc in Computer Science from University of Lagos in 1997 and a Ph.D in Computer Science in 2003 from the university of Agriculture, Abeokuta. His research interest includes Adoption of Information Systems strategies, Human Computer Interactions (HCI), Knowledge Management, Image Processing and Computational Intelligence . He is a member of Nigeria Computer Society and Computer Professional Registration Council of Nigeria. He has published in reputable international and local Journals. 